Discussion Paper No. 683

\title{
QUEUEING PROBLEMS WITH \\ TWO PARALLEL SERVERS
}

\author{
Youngsub Chun \\ and \\ Eun Jeong Heo
}

\begin{abstract}
March 2007
The Institute of Social and Economic Research Osaka University

6-1 Mihogaoka, Ibaraki, Osaka 567-0047, Japan
\end{abstract}




\title{
Queueing Problems with Two Parallel Servers
}

\author{
Youngsub Chun* Eun Jeong Heo ${ }^{\dagger}$
}

February 2007

\begin{abstract}
A group of agents are waiting for their job to be processed in a facility. We assume that each agent needs the same amount of processing time and incurs waiting costs. The facility has two parallel servers, being able to serve two agents at a time. We are interested in finding the order to serve agents and the (positive or negative) monetary compensations they should receive. We introduce two rules for the problem, the minimal transfer rule and the maximal transfer rule. We show that these two rules correspond to the Shapley (1953) value of the queueing games with two servers, as discussed similarly by Maniquet (2003) and Chun (2006a) for queueing problems with one serve, when the worth of each coalition is appropriately defined. If the worth of a coalition is defined by assuming the coalitional members are served before the non-coalitional members, then the minimal transfer rule is obtained. On the other hand, if it is defined by assuming the coalitional members are served after the non-coalitional members, then the maximal transfer rule is obtained.
\end{abstract}

Journal of Economic Literature Classification Numbers: C71, D63, D73.

Keywords: queueing problems with two servers, Shapley value, minimal transfer rule, maximal transfer rule.

${ }^{*}$ School of Economics, Seoul National University, Seoul, 151-746, Korea, E-mail: ychun@snu.ac.kr.

${ }^{\dagger}$ Department of Economics, University of Rochester, Rochester NY 14627, USA, Email: eheo@troi.cc.rochester.edu. We are grateful to Sunghoon Hong for his comments. This work was supported by the Second Brain Korea 21 project in 2006. 


\section{Introduction}

The queueing problem is concerned with the following situation. A group of agents are waiting for their job to be processed in a facility. The facility can handle one agent at a time, and each agent needs the same amount of time for the job to be processed. Furthermore, an agent's waiting cost is assumed to be constant per unit of time, but agents differ in their waiting costs. Each agent's utility is equal to the amount of his monetary transfer minus his total waiting costs. We are interested in finding the order in which to serve agents and the (positive or negative) monetary compensations they should receive. Recently, this problem has been analyzed in various perspectives: incentive issues (Dolan, 1978; Suijs, 1996; Mitra 2001, 2002), cooperative game theoretic approach (Maniquet, 2003; Chun, 2006a; Chun and Hokari, 2004), no-envy (Chun, 2006b), merging and splitting (Moulin, 2004, 2006; Özsoy 2005), and others.

In particular, Maniquet (2003) proposes the minimal transfer rule for a model in which a facility serves one agent at a time. This rule corresponds to the Shapley (1953) value of the game when the worth of each coalition is defined to be the minimum waiting costs incurred by its members under the optimistic assumption that they are served before the non-coalitional members. On the other hand, Chun (2006a) proposes the maximal transfer rule and shows that it corresponds to the Shapley value of the game when the worth of each coalition is defined to be the minimum waiting costs incurred by its members under the pessimistic assumption that they are served after the non-coalitional members.

In this paper, we extend the model by assuming the facility has two parallel servers. Also, we introduce two rules for the problem, the minimal and the maximal transfer rule, and discuss their properties. As in Maniquet (2003) and Chun (2006a), our rules correspond to the Shapley value of the game in which the worth of each coalition is appropriately defined. However, our results are not a simple adaptation of previous results since we need to consider the transfer between two agents served at the same time. According 
to our rules, even though two agents are served at the same time, their transfers are different: the transfer can be interpreted as if an agent with a smaller unit waiting cost receives a monetary transfer from the other agent in the same group with a larger unit waiting cost. If an agent preceding the group leaves, then the agent with a larger unit waiting cost moves to the preceding group, thus saving a unit waiting cost. Such a contingent possibility requires that the monetary transfer should be imposed agents in the same group as well as those in the different groups.

We begin our study in the hope to give a sufficient insight to deal with the queueing problem with an arbitrary number of servers. However, we could not achieve our goal yet. We can provide an answer to problems with two parallel servers, but it cannot be generalized to problems with more than two servers due to computational difficulties.

The paper is organized as follows. Section 2 presents preliminaries. Section 3 discusses how the cooperative game theory can be applied to solve our queueing problems and investigates implications of the optimistic approach. Section 4 investigates implications of the pessimistic approach in the queueing problems with two servers. In section 5 , we conclude by discussing other possible extensions of the problem.

\section{Preliminaries}

Let $I \equiv\{1,2, \cdots\}$ be an (infinite) universe of "potential" agents, and $\mathcal{N}$ be the family of non-empty finite subsets of $I$. Each agent $i \in I$ is characterized by his unit waiting cost, $\theta_{i} \geq 0$. We assume that the facility can handle two agents at a time and each agent needs the same amount of service time, normalized as unit time. Given $N \in \mathcal{N}$, each agent $i \in N$ is assigned a group $g_{i} \in \mathbf{N}$ which is his service order in the queue, and a positive or negative transfer $t_{i} \in \mathbb{R}$. The group which is served first incurs no waiting cost. If agent $i \in N$ is served in the $g_{i}$ th group, his waiting cost is $\left(g_{i}-1\right) \theta_{i}$. Each agent $i \in N$ has a quasi-linear utility function: his utility from the bundle $\left(g_{i}, t_{i}\right)$ is given by $u\left(g_{i}, t_{i} ; \theta_{i}\right)=t_{i}-\left(g_{i}-1\right) \theta_{i}$. 
A queueing problem with two parallel servers or a two-server queueing problem is defined as a list $q=(N, \theta ; 2)$ where $N \in \mathcal{N}$ is the set of agents, $\theta \in \mathbb{R}_{+}^{N}$ is the vector of unit waiting costs, and 2 is the number of servers. Since we analyze two-server problems, 2 indicating the number of servers is omitted for simplicity. Let $\mathcal{Q}^{N}$ be the class of all two-server queueing problems for $N$ and $\mathcal{Q}=\cup \mathcal{Q}^{N}$. An allocation for $q \in \mathcal{Q}$ is a pair $z=(g, t)$, where for each $i \in N, g_{i}$ the group to which agent $i$ belongs, and $t_{i}$ the monetary transfer to him. An allocation is feasible if less than or equal to two agents are assigned to each group and the sum of transfers is not positive. Thus, the set of feasible allocations $Z(q)$ consists of all pairs $z=(g, t)$ such that for any distinct agents $i, j, k \in N, g_{i}=g_{j}$ implies $g_{k} \neq g_{i}$ and $\sum_{i \in N} t_{i} \leq$ 0 .

Given $q=(N, \theta) \in \mathcal{Q}^{N}$, an allocation $z=(g, t) \in Z(q)$ is group efficient if it minimizes the total waiting costs, that is, for each $z^{\prime}=\left(g^{\prime}, t^{\prime}\right) \in Z(q)$, $\sum_{i \in N}\left(g_{i}-1\right) \theta_{i} \leq \sum_{i \in N}\left(g_{i}^{\prime}-1\right) \theta_{i}$. The efficient group of a problem does not depend on the transfers. Moreover, it is unique except for agents with equal unit waiting costs. The set of efficient groups for $q \in \mathcal{Q}^{N}$ is denoted by $\operatorname{Eff}(q)$. For a group to be efficient, two agents with the largest unit waiting costs should be in the first group, and the two agents with the next two largest unit waiting cost should be in the second group, and so on.

Given $q=(N, \theta) \in \mathcal{Q}^{N}$, an allocation $z=(g, t) \in Z(q)$ is budget balanced if $\sum_{i \in N} t_{i}=0$. An allocation rule, or simply a rule, is a mapping $\varphi: \mathcal{Q} \rightarrow$ $\sum_{N \in \mathcal{N}} Z(q)$, which associates with every $N \in \mathcal{N}$ and every $q \in \mathcal{Q}^{N}$ a nonempty subset $\varphi(q)$ of feasible allocations. The pair $\varphi_{i}(q)=\left(g_{i}, t_{i}\right)$ represents the service time of agent $i$ and his transfer in $q$ under $\varphi$.

To facilitate our analysis, we rename agents by index $d$ according to their unit waiting costs. We assign an agent with the largest unit waiting cost 1 , the second largest 2, and so on. The index is unique except for agents with equal waiting costs. These agents have to be indexed consecutively but in any order. Given $q=(N, \theta) \in \mathcal{Q}^{N}$, let $D(q)$ be the set of all possible indices. If we line up agents according to the index, and assign the first two agents to 
the first group, the next two agents to the second group, and so on, we have an efficient group. That is, for each $d \in D(q)$ and each $i \in N, g_{i}$ is defined as

$$
g_{i}=\left\lceil\frac{d_{i}}{2}\right\rceil=\left\{\begin{array}{cl}
\frac{d_{i}}{2} & \text { if } d_{i} \text { is even } \\
\frac{d_{i}+1}{2} & \text { if } d_{i} \text { is odd }
\end{array}\right.
$$

Then, the resulting group is efficient. Given $q=(N, \theta) \in \mathcal{Q}^{N}, d \in D(q)$, and $i \in N$, let $P_{i}(d)$ be the set of agents with smaller indices than agent $i$ and $F_{i}(d)$ the set of agents with larger indices than agent $i$.

Now we define the minimal transfer rule. This rule chooses an efficient group. Then, the transfer to agent $i$ is assigned as if it consists of two parts, compensation to agent $i$ and payment of agent $i$ : compensation to agent $i$ is the sum of all the group numbers assigned to all agents in the preceding groups divided by $i$ 's index. After determining compensations to each agent, the compensation is assigned equally to agents with smaller indices as payment. Thus, the payment of agent $i$ is the sum of all those assigned to agent $i$ from all other agents with larger indices.

Minimal transfer rule: for each $N \in \mathcal{N}$, each $q \in \mathcal{Q}^{N}$, and each $d \in D(q)$,

$$
\begin{aligned}
\varphi^{M}(q)=\left\{\left(g^{M}, t^{M}\right) \in Z(q) \mid g^{M} \in \operatorname{Eff}(q), \text { and } \forall i \in N,\right. \\
t_{i}^{M}=\frac{\sum_{g_{j}^{M}<g_{i}^{M}} g_{j}^{M} \cdot 2}{d_{i}} \cdot \theta_{i}-\sum_{k \in F_{i}(d)}\left\{\frac{1}{d_{k}-1} \cdot \frac{\sum_{g_{j}^{M}<g_{k}^{M}} g_{j}^{M} \cdot 2}{d_{k}} \cdot \theta_{k}\right\} .
\end{aligned}
$$

Remark 1: Alternatively, the transfer can be expressed as follows. For each $i \in N$,

$$
t_{i}^{M}=\frac{g_{i}^{M}\left(g_{i}^{M}-1\right)}{d_{i}} \theta_{i}-\sum_{j \in F_{i}(d)} \frac{1}{d_{j}-1} \frac{g_{j}^{M}\left(g_{j}^{M}-1\right)}{d_{j}} \theta_{j} .
$$

Since this expression is somewhat easier to manipulate, we will use it in the proofs.

Remark 2: We show that our minimal transfer rule coincides with the rule discussed in Maniquet (2003) for one-server problems, in which a facility can 
handle one agent at a time. Since only one agent is served in one-server problems at a time, group $g_{i}$ can be replaced by index $d_{i}$ which represents $i$ 's position in the queue, and 2 in the numerator of the compensation should be changed to 1 . Altogether, the definition becomes

$$
\begin{aligned}
t_{i}^{M}\left(q_{1}\right) & =\frac{\sum_{d_{j}<d_{i}} d_{j} \cdot 1}{d_{i}} \cdot \theta_{i}-\sum_{k \in N: d_{k}>d_{i}}\left(\frac{1}{d_{k}-1} \cdot \frac{\sum_{d_{j}<d_{k}} d_{j} \cdot 1}{d_{k}} \cdot \theta_{k}\right) \\
& =\frac{d_{i}-1}{2} \theta_{i}-\sum_{k \in N: d_{k}>d_{i}} \frac{1}{2} \theta_{k},
\end{aligned}
$$

as desired. Moreover, as discussed in Section 3, our rule can be characterized by axioms in the same spirit as Maniquet's (2003).

Next we present an example to show how the minimal transfer rule is calculated.

Example 1: Minimal transfer rule. Let $N \equiv\{1,2,3,4,5,6\}$ and $\theta \equiv$ $\left(\theta_{1}, \theta_{2}, \theta_{3}, \theta_{4}, \theta_{5}, \theta_{6}\right)$ be such that $\theta_{1}>\theta_{2}>\theta_{3}>\theta_{4}>\theta_{5}>\theta_{6}$. By efficiency, $g^{M}=(1,1,2,2,3,3)$ and $d=(1,2,3,4,5,6)$. The transfer assigned by the minimal transfer rule is

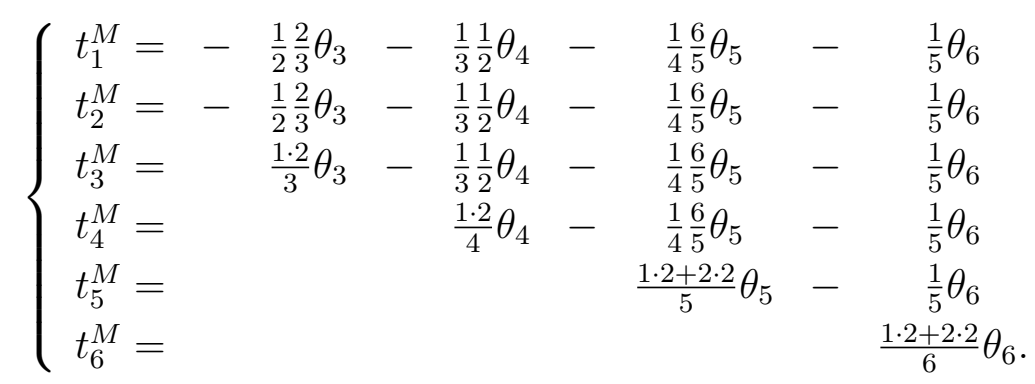

Since agents 3 and 4 belong to the same group, they are served at the same time. However, the minimal transfer rule assigns different amount of transfers to them. In fact, agent 3's transfer has the term $-(1 / 6) \theta_{4}$, which can be regarded as payment of 3 to 4 . If either agent 1 or agent 2 leaves the queue without being served, then one agent in the second group moves up to the first group. By efficiency of the minimal transfer rule, an agent with a larger unit waiting cost, agent 3, moves up. Since agent 3 can save his waiting cost in the process, agent 3 compensates to agent 4 even though they are served at the same time now. 


\section{An optimistic approach to queueing games}

The two-server queueing problem can solved by applying cooperative game theoretic solution as in Maniquet (2003) and Chun (2006a). First, we formally describe TU (transferable utility) games. Let the set of players $N \in \mathcal{N}$ be given. A set $S \subseteq N$ is a coalition. A TU game, or a game, is a real-valued function $v$ defined on all coalitions $S \subseteq N$ satisfying $v(\emptyset)=0$. The number $v(S)$ represents the worth of a coalition $S$. Let $\Gamma^{N}$ be the class of games with player set $N$, and $\Gamma=\cup \Gamma^{N}$. A value is a function $\phi$ defined on $\Gamma$ which associates with every $N \in \mathcal{N}$ and every $v \in \Gamma^{N}$ a vector $\phi(v)=\left(\phi_{i}(v)\right)_{i \in N}$. The number $\phi_{i}(v)$ is the payoff to player $i$ in game $v$.

We introduce the best-known value for games, the Shapley (1953) value: it assigns each player a payoff equal to a weighted average of his marginal contributions to all possible coalitions, with weights being determined by the size of coalitions.

Shapley value, SV: For each $q=(N, \theta) \in \mathcal{Q}^{N}$ and each $i \in N$,

$$
S V_{i}(v)=\sum_{S: i \in S} \frac{(|S|-1) !(|N \backslash S|) !}{|N| !}\{v(S)-v(S \backslash\{i\})\} .
$$

As in Maniquet (2003), we define the worth of each coalition $S \subseteq N$ to be the minimum waiting cost incurred by its members assuming that they are served before the non-coalitional members. That is, for each $S \subseteq N$, its worth $v^{M}(S)$ is defined by setting

$$
v^{M}(S)=-\sum_{i \in S}\left(g_{i}^{S}-1\right) \theta_{i},
$$

where $g^{S} \in \operatorname{Eff}\left(q^{S}\right)$ and $q^{S}=\left(S,\{\theta\}_{i \in S}\right)$.

Next is an example showing that the Shapely value applied to the queueing game results in the same payoff as our minimal transfer rule.

Example 2: (Example 1 continued.) Let $N$ and $\theta$ be defined as in Example 1. The Shapley value assigns each agent the following payoff: $S V\left(v^{M}\right)=$ 
$\left(-\frac{1}{3} \theta_{3}-\frac{1}{6} \theta_{4}-\frac{3}{10} \theta_{5}-\frac{1}{5} \theta_{6},-\frac{1}{3} \theta_{3}-\frac{1}{6} \theta_{4}-\frac{3}{10} \theta_{5}-\frac{1}{5} \theta_{6},-\frac{1}{3} \theta_{3}-\frac{1}{6} \theta_{4}-\frac{3}{10} \theta_{5}-\right.$ $\left.\frac{1}{5} \theta_{6},-\frac{1}{2} \theta_{4}-\frac{3}{10} \theta_{5}-\frac{1}{5} \theta_{6},-\frac{4}{5} \theta_{5}-\frac{1}{5} \theta_{6},-\theta_{6}\right)$.

Since $t_{i}=\left(g_{i}-1\right) \theta_{i}+u_{i}$, the corresponding transfer is $t=\left(-\frac{1}{3} \theta_{3}-\frac{1}{6} \theta_{4}-\right.$ $\frac{3}{10} \theta_{5}-\frac{1}{5} \theta_{6},-\frac{1}{3} \theta_{3}-\frac{1}{6} \theta_{4}-\frac{3}{10} \theta_{5}-\frac{1}{5} \theta_{6}, \frac{2}{3} \theta_{3}-\frac{1}{6} \theta_{4}-\frac{3}{10} \theta_{5}-\frac{1}{5} \theta_{6}, \frac{1}{2} \theta_{4}-\frac{3}{10} \theta_{5}-$ $\frac{1}{5} \theta_{6}, \frac{6}{5} \theta_{5}-\frac{1}{5} \theta_{6}, \theta_{6}$ ), which coincides with the transfer assigned by the minimal transfer rule in Example 1.

Before we discuss the relation between the minimal transfer rule and the Shapley value, we show how the dividend can be calculated for two-server queueing games. It is well-known that a TU-game $v$ can be written as a linear combination of unanimity games, that is, $v=\sum_{T \subseteq N} \lambda_{v}(T) u_{T}$, where the unanimity game $u_{T}$ on $N$ is given by $u_{T}(S)=1$ if $T \subseteq S$, and $u_{T}=0$ otherwise. For each $S \subseteq N$, its dividend $\lambda_{v}(S)$ is defined by if $|S|=1$, then $\lambda_{v}(S)=v(S)$, and if $|S|>1, \lambda_{v}(S)=v(S)-\sum_{T \nsubseteq S} \lambda_{v}(T)$.

Lemma 1. For each $q=(N, \theta) \in \mathcal{Q}^{N}$, the unanimity coefficient of $S \subseteq N$ is defined as

$$
\lambda_{v^{M}}(S)=\left\{\begin{array}{cl}
0 & \text { if }|S|=1 \text { or } 2 \\
-(-2)^{|S|-3} \min _{k \in S} \theta_{k} & \text { if }|S| \geq 3 .
\end{array}\right.
$$

Proof. If $|S|=1$ or 2 , the conclusion is obtained trivially from $v^{M}(S)=$ 0 . If $|S|=3, \lambda_{v^{M}}(S)=v^{M}(S)-\sum_{T \subsetneq S} \lambda_{v^{M}}(T)=v^{M}(S)=-\min _{i \in S} \theta_{i}=$ $-(-2)^{3-3} \min _{i \in S} \theta_{i}$, as desired. Now, as induction hypothesis, suppose that the conclusion holds for all $S \subset N$ such that $|S| \leq s-1$. We need to show that the conclusion is true for $|S|=s$. Without loss of generality, we may assume that $S \equiv\{1,2, \ldots, s\}, \theta_{1} \geq \theta_{2} \geq \cdots \geq \theta_{s}$, and that for each $i \in S, d_{i}=i$. Thus, for each $i \in S$, if $i$ is odd, $g_{i}=(i+1) / 2$, and if $i$ is even, $g_{i}=i / 2$. We will use the binomial theorem, $(a+b)^{n}=\sum_{i=0}^{n}\left(\begin{array}{l}n \\ i\end{array}\right) a^{i} b^{n-i}$ after replacing $a$ with -2 and $b$ with 1 . First, we rewrite $\lambda_{v^{M}}(S)$ :

$$
\begin{aligned}
\lambda_{v^{M}}(S) & =v^{M}(S)-\sum_{T \subsetneq S} \lambda_{v^{M}}(T) \\
& =v^{M}(S)+\sum_{T \subsetneq S,|T| \geq 3}(-2)^{|T|-3} \min _{i \in T} \theta_{i} \\
& =v^{M}(S)+\sum_{i=3}^{s-1}\left(\sum_{j=3}^{i}\left(\begin{array}{c}
i-1 \\
j-1
\end{array}\right)(-2)^{j-3}\right) \theta_{i}+\sum_{j=3}^{s-1}\left(\begin{array}{c}
s-1 \\
j-1
\end{array}\right)(-2)^{j-3} \theta_{s}
\end{aligned}
$$


By the binomial theorem and the definition of $g_{i}$, the second term is rewritten as:

$$
\begin{aligned}
& \sum_{i=3}^{s-1}\left(\sum_{j=3}^{i}\left(\begin{array}{c}
i-1 \\
j-1
\end{array}\right)(-2)^{j-3}\right) \theta_{i} \\
= & \sum_{i=3}^{s-1}\left(\sum_{j=2}^{i-1}\left(\begin{array}{c}
i-1 \\
j
\end{array}\right)(-2)^{j-2}\right) \theta_{i} \\
= & \sum_{i=3}^{s-1} \frac{1}{4}\left(\sum_{j=2}^{i-1}\left(\begin{array}{c}
i-1 \\
j
\end{array}\right)(-2)^{j}(1)^{i-1-j}\right) \theta_{i} \\
= & \sum_{i=3}^{s-1} \frac{1}{4}\left[\left(\sum_{j=0}^{i-1}\left(\begin{array}{c}
i-1 \\
j
\end{array}\right)(-2)^{j}(1)^{i-1-j}\right)-1+2(i-1)\right] \theta_{i} \\
= & \sum_{i=3}^{s-1} \frac{1}{4}\left[(-2+1)^{i-1}-1+2(i-1)\right] \theta_{i} \\
= & \sum_{i=3}^{s-1} \frac{(-1)^{i-1}-1+2(i-1)}{4} \theta_{i} \\
= & \sum_{i=3}^{s-1}\left(g_{i}-1\right) \theta_{i} .
\end{aligned}
$$

Similarly, the third term is rewritten as:

$$
\begin{aligned}
& \sum_{j=3}^{s-1}\left(\begin{array}{c}
s-1 \\
j-1
\end{array}\right)(-2)^{j-3} \theta_{s} \\
= & \sum_{j=2}^{s-2}\left(\begin{array}{c}
s-1 \\
j
\end{array}\right)(-2)^{j-2} \theta_{s} \\
= & \sum_{j=2}^{s-1}\left(\begin{array}{c}
s-1 \\
j
\end{array}\right)(-2)^{j-2} \theta_{s}-(-2)^{s-3} \theta_{s} \\
= & \frac{1}{4} \sum_{j=2}^{s-1}\left(\begin{array}{c}
s-1 \\
j
\end{array}\right)(-2)^{j} \theta_{s}-(-2)^{s-3} \theta_{s} \\
= & \frac{1}{4}\left[\sum_{j=0}^{s-1}\left(\begin{array}{c}
s-1 \\
j
\end{array}\right)(-2)^{j}-1+2(s-1)\right] \theta_{s}-(-2)^{s-3} \theta_{s} \\
= & \frac{1}{4}\left[(-2+1)^{s-1}-1+2(s-1)\right] \theta_{s}-(-2)^{s-3} \theta_{s} \\
= & \frac{(-1)^{s-1}-1+2(s-1)}{4} \theta_{s}-(-2)^{s-3} \theta_{s} \\
= & \left(g_{s}-1\right) \theta_{s}-(-2)^{s-3} \theta_{s} .
\end{aligned}
$$

Since $v^{M}(S)=-\sum_{i=3}^{s}\left(g_{i}-1\right) \theta_{i}, \lambda_{v^{M}}(S)=-(-2)^{s-3} \theta_{s}=-(-2)^{s-3} \min _{i \in S} \theta_{i}$, the desired conclusion.

Now we prove that the minimal transfer rule assigns the same payoff as the Shapley value applied to the queueing game when the worth of a coalition is optimistically defined.

Theorem 1. Let $q \in \mathcal{Q}^{N}$ and $d \in D(q)$. Let $z=(g, t) \in Z(q)$ be such that agents' utilities at $z$ are equal to the payoff vector obtained by applying the Shapley value to $v^{M}$. Then, $g \in E f f(q)$ and for each $i \in N, t_{i}=t_{i}^{M}$. 
Proof. Let $N \equiv\{1,2, \ldots, n\}$. Without loss of generality, we may assume that $\theta_{1} \geq \theta_{2} \geq \cdots \geq \theta_{n}$ and that for each $i \in N, d_{i}=i$. By Lemma 1 , for each $i \in N$, the allocation assigned by the Shapley value can be calculated as follows:

$$
\begin{aligned}
S V_{i}\left(v^{M}\right) & =\sum_{i \in S, S \subseteq N} \frac{\lambda_{v M}(S)}{|S|} \\
& =\sum_{k=3}^{i} \frac{-(-2)^{k-3}}{k}\left(\begin{array}{c}
i-1 \\
k-1
\end{array}\right) \theta_{i}+\sum_{j=i+1}^{n} \sum_{k=3}^{j} \frac{-(-2)^{k-3}}{k}\left(\begin{array}{c}
j-2 \\
k-2
\end{array}\right) \theta_{j}
\end{aligned}
$$

By the binomial theorem and the definition of $g_{i}$, the first term in $S V_{i}\left(v^{M}\right)$ can be rewritten as: for each $i \in N$,

$$
\begin{aligned}
& \sum_{k=3}^{i} \frac{-(-2)^{k-3}}{k}\left(\begin{array}{c}
i-1 \\
k-1
\end{array}\right) \theta_{i} \\
= & \sum_{k=3}^{i} \frac{-(-2)^{k-3}}{k} \frac{(i-1) !}{(i-k) !(k-1) !} \theta_{i} \\
= & \sum_{k=3}^{i}-(-2)^{k-3} \frac{i !}{(i-k) ! k !} \frac{1}{i} \theta_{i} \\
= & \sum_{k=3}^{i} \frac{-(-2)^{k-3}}{i}\left(\begin{array}{l}
i \\
k
\end{array}\right) \theta_{i} \\
= & \frac{1}{8 i} \sum_{k=3}^{i}(-2)^{k}\left(\begin{array}{l}
i \\
k
\end{array}\right) \theta_{i} \\
= & \frac{1}{8 i}\left[\sum_{k=0}^{i}(-2)^{k}\left(\begin{array}{l}
i \\
k
\end{array}\right)-\left(1-2 i+4 \frac{(i-1) i}{2}\right)\right] \theta_{i} \\
= & \frac{1}{8 i}\left[(-1)^{i}-\left(1-2 i+4 \frac{(i-1) i}{2}\right)\right] \theta_{i} \\
= & \frac{1}{8 i}\left((-1)^{i}-1+2 i-2 i(i-1)\right) \theta_{i} \\
= & {\left[-\left(g_{i}-1\right)+\frac{g_{i}\left(g_{i}-1\right)}{i}\right] \theta_{i} . }
\end{aligned}
$$

Similarly, by (1) and (2), the second term in $S V_{i}\left(v^{M}\right)$ can be rewritten as:

$$
\begin{aligned}
& \sum_{j=i+1}^{n} \sum_{k=3}^{j} \frac{-(-2)^{k-3}}{k}\left(\begin{array}{l}
j-2 \\
k-2
\end{array}\right) \theta_{j} \\
= & \sum_{j=i+1}^{n} \sum_{k=3}^{j} \frac{-(-2)^{k-3}}{k} \frac{k-1}{j-1}\left(\begin{array}{l}
j-1 \\
k-1
\end{array}\right) \theta_{j} \\
= & -\sum_{j=i+1}^{n} \frac{1}{j-1} \sum_{k=3}^{j}(-2)^{k-3} \frac{k-1}{k}\left(\begin{array}{l}
j-1 \\
k-1
\end{array}\right) \theta_{j} \\
= & \left.-\sum_{j=i+1}^{n} \frac{1}{j-1}\left[\sum_{k=3}^{j}(-2)^{k-3}\left(\begin{array}{l}
j-1 \\
k-1
\end{array}\right)-\sum_{k=3}^{j} \frac{(-2)^{k-3}}{k}\left(\begin{array}{l}
j-1 \\
k-1
\end{array}\right)\right)\right] \theta_{j} \\
= & -\sum_{j=i+1}^{n} \frac{1}{j-1}\left[\left(g_{j}-1\right)-\left(g_{j}-1\right)+\frac{\left(g_{j}-j\right)\left(g_{j}-1\right)}{j}\right] \theta_{j} \\
= & -\sum_{j=i+1}^{n} \frac{1}{j-1} \frac{g_{j}\left(g_{j}-1\right)}{j} \theta_{j} .
\end{aligned}
$$

Altogether, for each $i \in N$, 


$$
\begin{aligned}
S V_{i}\left(v^{M}\right) & =\sum_{k=3}^{i} \frac{-(-2)^{k-3}}{k}\left(\begin{array}{c}
i-1 \\
k-1
\end{array}\right) \theta_{i}+\sum_{j=i+1}^{n} \sum_{k=3}^{j} \frac{-(-2)^{k-3}}{k}\left(\begin{array}{c}
j-2 \\
k-2
\end{array}\right) \theta_{j} \\
& =\left[-\left(g_{i}-1\right)+\frac{g_{i}\left(g_{i}-1\right)}{i}\right] \theta_{i}-\sum_{j=i+1}^{n} \frac{1}{j-1} \frac{g_{j}\left(g_{j}-1\right)}{j} \theta_{j}
\end{aligned}
$$

which is the desired expression as noted in Remark 1.

Remark 3: The minimal transfer rule can be characterized by axioms used in Maniquet (2003), but appropriately modified to be suitable for two-server queueing problems. In fact, (1) it is the only rule satisfying efficiency, Pareto indifference, equal treatment of equals, and independence of larger costs; (2) it is the only rule satisfying Pareto indifference, the identical preferences lower bound, negative cost monotonicity, and least-cost agent equal responsibility; (3) it minimizes the sum of the absolute values of transfers among agents among the rules satisfying Pareto indifference, the identical preferences lower bound, and least-cost agent equal responsibility. ${ }^{1}$

\section{A pessimistic approach and the maximal transfer rule}

Now we investigate a pessimistic definition for a worth of a coalition which assumes the members in a coalition are served after the non-coalitional members. As in Chun (2006a), even though we apply the same Shapley value to the game, the resulting rule is different.

\footnotetext{
${ }^{1}$ Efficiency requires that the rule should choose allocations that are group efficient and budget balanced. Pareto indifference requires that if an allocation is chosen by a rule, then all other allocations which assign the same utilities to each agent should be chosen by the rule. Equal treatment of equals requires that those who have the same unit waiting cost should end up with the same utilities. Independence of larger costs requires that an increase in an agent's unit waiting cost should not affect agents with smaller unit waiting costs. The identical cost lower bound (Moulin, 1990) requires that each agent should be at least as well off as he would be, under efficiency and equal treatment of equals, if all agents had the same unit waiting cost. Negative cost monotonicity requires that an increase in an agent's unit waiting cost should cause all other agents to weakly lose. Finally, least-cost agent equal responsibility requires that upon the departure of the agent served last, the rule should choose a group efficient allocation which divides the last agent's transfer equally among all remaining agents.
} 
First, we introduce an alternative definition for a worth of a coalition $S \subseteq N$ from the pessimistic viewpoint. To do this, we need to consider the cardinality of $N \backslash S$ because it is served before the coalition $S$. If $|N \backslash S|$ is even, then agents in $S$ will be served from $\left(\frac{|N|-|S|}{2}+1\right)$ th group. If $|N \backslash S|$ is odd, the last group of $N \backslash S$ is composed of one agent from $N \backslash S$ and one agent from $S$. Therefore, the waiting cost of $i \in S, C_{i}(S)$, can be calculated as follows. For each $i \in S$,

$$
C_{i}(S)= \begin{cases}\left(\frac{|N|-|S|}{|2|}+\left(g_{i}^{S}-1\right)\right) \theta_{i} & \text { if }|N|-|S| \text { is even, } \\ \left(\frac{|N|-|S|-1}{|N|-|S|-1}+\left(g_{i}^{S}-1\right)\right) \theta_{i} & \text { if }|N|-|S| \text { is odd and } d_{i}^{S} \text { is odd } \\ \left(\frac{|N|-\mid S}{2}+g_{i}\right. & \text { if }|N|-|S| \text { is odd and } d_{i}^{S} \text { is even. }\end{cases}
$$

where $g^{S} \in \operatorname{Eff}\left(q^{S}\right)$ and $q^{S}=\left(S,\{\theta\}_{i \in S}\right)$. Also, the worth of a coalition $S$ from the pessimistic viewpoint, $v^{X}(S)$, is defines as

$$
v^{X}(S)=-\sum_{i \in S} C_{i}(S)
$$

Next we introduce the maximal transfer rule. This rule chooses an efficient group. Once again, the transfer to agent $i$ is assigned as if it consists of two parts, compensation to agent $i$ and payment of agent $i$. Assuming an even number of agents, payment of agent $i$ is the sum of all the group numbers assigned to following groups minus $i$ 's group number times 2 (which is the number of agents in the group) divided by the followers including him. After determining payments to each agent, the amount is assigned equally to agents with larger indices than agent $i$ as compensation. Thus, the compensation to agent $i$ is the sum of all those assigned to agent $i$ from all other agents with smaller indices.

We note that there is a symmetry between the minimal and the maximal transfer rules. The preceding groups are considered in calculating the "compensation" for the minimal transfer rule, and then the preceding agents pay the compensation equally. On the other hand, following groups are considered in calculating the "payment" for the maximal transfer rule, and then the following agents share the payment equally. 
For each $N \in \mathcal{N}$, each $q \in \mathcal{Q}^{N}$, each $d \in D(q)$, and each $i \in N$, let $p_{i}$ be the payment of agent $i$, defined as

$$
p_{i}= \begin{cases}\frac{\sum_{g_{j}>g_{i}}\left(g_{j}-g_{i}\right) \cdot 2}{n-d_{i}+1} \theta_{i} & \text { if } n \text { is even } \\ \frac{\left.\left.\sum_{g_{j}>g_{i}, g_{j}<\left\lceil\frac{n}{2}\right\rceil}\right\rceil g_{j}-g_{i}\right) \cdot 2}{n-d_{i}+1} \theta_{i}+\frac{\left\lceil\frac{n}{2}\right\rceil-g_{i}}{n-d_{i}+1} \theta_{i} & \text { if } n \text { is odd }\end{cases}
$$

Maximal transfer rule: for each $N \in \mathcal{N}$, each $q \in \mathcal{Q}^{N}$, and each $d \in D(q)$,

$$
\begin{aligned}
\varphi^{X}(q)= & \left\{\left(g^{X}, t^{X}\right) \in Z(q) \mid g^{X} \in E f f(q), \text { and } \forall i \in N\right. \\
& \left.t_{i}^{X}=\sum_{j \in P_{i}(d)} \frac{p_{j}}{n-d_{j}}-p_{i}\right\} .
\end{aligned}
$$

Next example shows how the transfer for the maximal transfer rule is calculated.

Example 3: The Shapley value in a pessimistic approach.

Let $N \equiv\{1,2,3,4,5,6\}$ and $\theta \equiv\left(\theta_{1}, \theta_{2}, \theta_{3}, \theta_{4}, \theta_{5}, \theta_{6}\right)$ be such that $\theta_{1}>$ $\theta_{2}>\theta_{3}>\theta_{4}>\theta_{5}>\theta_{6}$. Now we apply the Shapley value in a pessimistic perspective and calculate the corresponding transfer.

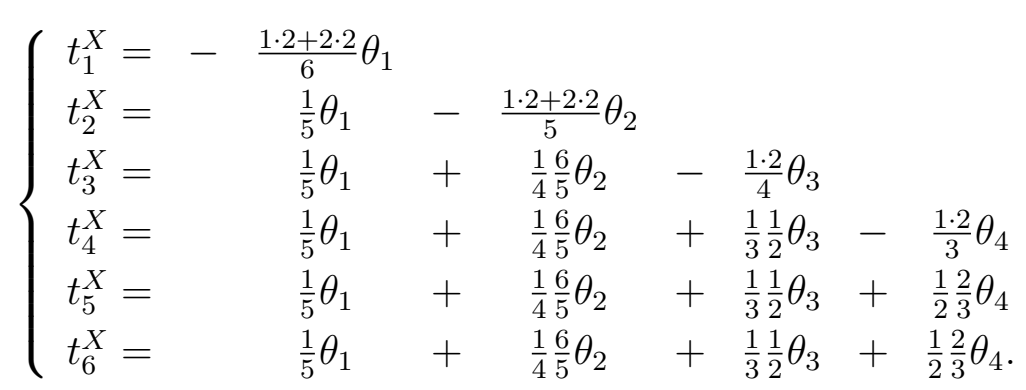

Therefore, it coincides with transfers specified by the maximal transfer rule.

We note that $p_{1}=\frac{\{(2-1)+(3-1)\} \cdot 2}{6-1+1} \theta_{1}=\theta_{1}$, and it is equally shared by the followers, $2,3,4,5$, and 6 , by $\frac{1}{5} \theta_{1}$. Similarly, $p_{2}=\frac{\{(2-1)+(3-1)\} \cdot 2}{6-2+1} \theta_{2}=\frac{6}{5} \theta_{2}$ and it is equally shared by the followers $3,4,5$, and 6 by $\frac{1}{4} \frac{6}{5} \theta_{2}$. And so on.

As in the optimistic approach, we relate the maximal transfer rule with the Shapley value. First, we derive the unanimity coefficient $\lambda_{v}$. 
Lemma 2. For each $q=(N, \theta) \in \mathcal{Q}^{N}$, the unanimity coefficient of $S \subseteq N$ is defined as follows. If $|N|$ is even, then

$$
\lambda_{v^{x}}(S)= \begin{cases}-\left(\left\lceil\frac{n}{2}\right\rceil-1\right) \theta_{i} & \text { if }|S|=1 \text { and } i \in S, \\ 0 & \text { if }|S|=2, \\ (-2)^{|S|-3} \max _{k \in S} \theta_{k} & \text { if }|S| \geq 3,\end{cases}
$$

and if $|N|$ is odd, then

$$
\lambda_{v^{x}}(S)= \begin{cases}-\left(\left\lceil\frac{n}{2}\right\rceil-1\right) \theta_{i} & \text { if }|S|=1 \text { and } i \in S \\ \max _{k \in S} \theta_{k} & \text { if }|S|=2 \\ -(-2)^{|S|-3} \max _{k \in S} \theta_{k} & \text { if }|S| \geq 3\end{cases}
$$

Proof. Let $N \equiv\{1,2, \ldots, n\}$. We will prove only when $|N|$ is even. For each $i \in N$, if $S=\{i\}$, then $\lambda_{v^{x}}(S)=v^{X}(S)=-\left(\left\lceil\frac{n}{2}\right\rceil-1\right) \theta_{i}$. For each $i$, $j \in N$ such that $i \neq j$, if $S=\{i, j\}$, then $\lambda_{v^{X}}(S)=v^{X}(S)-\sum_{T \subsetneq S} \lambda_{v^{X}}(T)=$ $-\left(\left\lceil\frac{n}{2}\right\rceil-1\right)\left(\theta_{i}+\theta_{j}\right)+\left(\left\lceil\frac{n}{2}\right\rceil-1\right) \theta_{i}+\left(\left\lceil\frac{n}{2}\right\rceil-1\right) \theta_{j}=0$. For each $i, j, k \in N$ such that $k>\max \{i, j\}$, if $S=\{i, j, k\}$, then $\lambda_{v^{x}}(S)=v^{X}(S)-\sum_{T \subsetneq S} \lambda_{v^{X}}(T)=$ $-\left(\left\lceil\frac{n}{2}\right\rceil-1\right)\left(\theta_{i}+\theta_{j}\right)-\left(\left\lceil\frac{n}{2}\right\rceil-2\right) \theta_{k}+\left(\left\lceil\frac{n}{2}\right\rceil-1\right)\left(\theta_{i}+\theta_{j}+\theta_{k}\right)=\theta_{k}$, as desired.

Now, as induction hypothesis, suppose that the conclusion holds for each $S \subset N$ such that $|S| \leq s-1$. We need to show that the conclusion is true for $|S|=s$. Without loss of generality, we assume that $S \equiv\{1,2, \ldots, s\}$ and that $\theta_{1} \leq \theta_{2} \leq \cdots \leq \theta_{s}$. First, we consider the case when $|S|$ is even.

$$
\begin{aligned}
\lambda_{v^{X}}(S)= & v^{X}(S)-\sum_{T \subsetneq S} \lambda_{v X}(T) \\
= & -\left(\left\lceil\frac{n}{2}\right\rceil-1\right)\left(\theta_{1}+\theta_{2}\right)-\left(\left\lceil\frac{n}{2}\right\rceil-2\right)\left(\theta_{3}+\theta_{4}\right)-\cdots-\left(\left\lceil\frac{n}{2}\right\rceil-\frac{s}{2}\right)\left(\theta_{s-1}+\theta_{s}\right) \\
& -\sum_{i=3}^{s-1}\left(\sum_{j=3}^{i}(-2)^{j-3}\left(\begin{array}{c}
i-1 \\
j-1
\end{array}\right)\right) \theta_{i}-\sum_{i=3}^{s-1}(-2)^{i-3}\left(\begin{array}{c}
i-1 \\
j-1
\end{array}\right) \theta_{s} \\
& +\left(\left\lceil\frac{n}{2}\right\rceil-1\right)\left(\theta_{1}+\theta_{2}+\cdots+\theta_{s}\right) \\
= & {\left[\left(\frac{s}{2}-1\right)\left(\theta_{s}+\theta_{s-1}\right)+\left(\frac{s}{2}-2\right)\left(\theta_{s-2}+\theta_{s-3}\right)+\cdots+\left(\theta_{4}+\theta_{3}\right)\right] } \\
& -\left[\sum_{i=3}^{s-1}\left(\sum_{j=3}^{i}(-2)^{j-3}\left(\begin{array}{c}
i-1 \\
j-1
\end{array}\right)\right) \theta_{i}\right]-\left[\sum_{i=3}^{s-1}(-2)^{i-3}\left(\begin{array}{c}
i-1 \\
j-1
\end{array}\right) \theta_{s}\right] .
\end{aligned}
$$

By the binomial theorem, the terms above can be rewritten as 


$$
\begin{aligned}
\sum_{i=3}^{s-1}\left(\sum_{j=3}^{i}(-2)^{j-3}\left(\begin{array}{c}
i-1 \\
j-1
\end{array}\right)\right) \theta_{i} & =\sum_{i=3}^{s-1} \frac{2 i-3+(-1)^{i-1}}{4} \theta_{i} \\
& =\left(\frac{s}{2}-1\right) \theta_{s-1}+\left(\frac{s}{2}-2\right)\left(\theta_{s-2}+\theta_{s-3}\right)+\cdots+\left(\theta_{4}+\theta_{3}\right) \\
\sum_{i=3}^{s-1}(-2)^{i-3}\left(\begin{array}{c}
s-1 \\
j-1
\end{array}\right) \theta_{s} & =\left[\frac{2 s-3+(-1)^{s-1}}{4}-(-2)^{s-3}\right] \theta_{s} \\
& =\left[\left(\frac{s}{2}-1\right)-(-2)^{s-3}\right] \theta_{s} .
\end{aligned}
$$

Altogether, $\lambda_{v^{x}}(S)=(-2)^{s-3} \theta_{s}=(-2)^{s-3} \max _{i \in S} \theta_{i}$, the desired conclusion.

The case when $|S|$ is odd can be proven in a similar way.

Now we show that the maximal transfer rule assigns the same payoff as the Shapley value applied to the queueing game when the worth of a coalition is pessimistically defined.

Theorem 2. Let $q \in \mathcal{Q}^{N}$ and $d \in D(q)$. Let $z=(g, t) \in Z(q)$ be such that agents' utilities at $z$ are equal to the payoff vector obtained by applying the Shapley value to $v^{X}$. Then, $g \in \operatorname{Eff}(q)$ and for each $i \in N, t_{i}=t_{i}^{X}$.

Proof. Let $N \equiv\{1,2, \ldots, n\}$. Without loss of generality, we may assume that $\theta_{1} \geq \theta_{2} \geq \cdots \geq \theta_{n}$ and that for each $i \in N, d_{i}=i$. First, we consider the case when $|N|$ is even. By Lemma 2, for each $i \in N$, the transfer assigned by the Shapley value can be expressed as:

$$
t_{i}=-\left(\left\lceil\frac{n}{2}\right\rceil-1\right) \theta_{i}+\sum_{i \in S, S \subseteq N} \frac{(-2)^{s-3} \max _{k \in S} \theta_{k}}{s}+\left(g_{i}-1\right) \theta_{i}
$$

where $|S|=s$. For each $i \in N, t_{i}$ can be rewritten as

$$
t_{i}=\left[\left(g_{i}-\left\lceil\frac{n}{2}\right\rceil\right)+\sum_{s=3}^{n-i+1} \frac{(-2)^{s-3}}{s}\left(\begin{array}{c}
n-i \\
s-1
\end{array}\right)\right] \theta_{i}+\sum_{j=1}^{i-1}\left[\sum_{s=3}^{n-j+1} \frac{(-2)^{s-3}}{s}\left(\begin{array}{c}
n-j-1 \\
s-2
\end{array}\right)\right] \theta_{j}
$$


By the binomial theorem, the terms in the first half are rewritten as

$$
\begin{aligned}
& \left(g_{i}-\left\lceil\frac{n}{2}\right\rceil\right)+\sum_{s=3}^{n-i+1} \frac{(-2)^{s-3}}{s}\left(\begin{array}{c}
n-i \\
s-1
\end{array}\right) \\
= & \left(g_{i}-\left\lceil\frac{n}{2}\right\rceil\right)+\sum_{s=3}^{n-i+1}(-2)^{s-3} \frac{(n-i+1) !}{(n-i-s+1) ! s !} \frac{1}{n-i+1} \\
= & \left(g_{i}-\left\lceil\frac{n}{2}\right\rceil\right)-\frac{1}{8(n-i+1)}\left\{\sum_{s=3}^{n-i+1}(-2)^{s}\left(\begin{array}{c}
n-i+1 \\
s
\end{array}\right)\right\} \\
= & \left(g_{i}-\left\lceil\frac{n}{2}\right\rceil\right)-\frac{1}{8(n-i+1)}\left[\sum_{s=0}^{n-i+1}(-2)^{s}\left(\begin{array}{c}
n-i+1 \\
s
\end{array}\right)-1+2(n-i+1)-2(n-i+1)(n-i)\right] \\
= & \left(g_{i}-\left\lceil\frac{n}{2}\right\rceil\right)+\frac{2(n-i+1)(n-i-1)+1-(-1)^{n-i+1}}{8(n-i+1)} \\
= & \left\{\begin{array}{ll}
-\frac{(n-i+2)(n-i)}{4(n-i+1)}, & \text { if } i \text { is even, } \\
-\frac{n-i-1}{4}, & \text { if } i \text { is odd, }
\end{array}\right\} \\
= & -\frac{\sum_{k=g_{i}+1}^{\left\lceil\frac{n}{2}\right\rceil}\left(k-g_{i}\right) \cdot 2}{n-i+1} .
\end{aligned}
$$

Also, by the binomial theorem and (3), the terms in the second half can be rewritten as

$$
\begin{aligned}
& \sum_{s=3}^{n-j+1} \frac{(-2)^{s-3}}{s}\left(\begin{array}{c}
n-j-1 \\
s-2
\end{array}\right) \\
= & \frac{1}{n-j}\left[(n-j) \sum_{s=3}^{n-j+1} \frac{(-2)^{s-3}}{s}\left(\begin{array}{c}
n-j-1 \\
s-2
\end{array}\right)\right] \\
= & \frac{1}{n-j}\left[\sum_{s=3}^{n-j+1}(-2)^{s-3} \frac{s-1}{n-j-s+1}\left(\begin{array}{c}
n-j \\
s
\end{array}\right)\right] \\
= & \frac{1}{n-j}\left[\sum_{s=3}^{n-j+1}(-2)^{s-3} \frac{s-1}{n-j-s+1}\left(\begin{array}{c}
n-j \\
s
\end{array}\right)+\sum_{s=3}^{n-j+1} \frac{(-2)^{s-3}}{s}\left(\begin{array}{c}
n-j \\
s-1
\end{array}\right)-\sum_{s=3}^{n-j+1} \frac{(-2)^{s-3}}{s}\left(\begin{array}{c}
n-j \\
s-1
\end{array}\right)\right] \\
= & \frac{1}{n-j}\left[\sum_{s=3}^{n-j+1}(-2)^{s-3}\left\{\frac{s-1}{n-j-s+1}\left(\begin{array}{c}
n-j \\
s
\end{array}\right)+\frac{1}{s}\left(\begin{array}{c}
n-j \\
s-1
\end{array}\right)\right\}-\sum_{s=3}^{n-j+1} \frac{(-2)^{s-3}}{s}\left(\begin{array}{c}
n-j \\
s-1
\end{array}\right)\right] \\
= & \frac{1}{n-j}\left[\sum_{s=3}^{n-j+1}(-2)^{s-3}\left(\begin{array}{c}
n-j \\
s-1
\end{array}\right)-\sum_{s=3}^{n-j+1} \frac{(-2)^{s-3}}{s}\left(\begin{array}{c}
n-j \\
s-1
\end{array}\right)\right] \\
= & \frac{1}{n-j}\left[\frac{1}{4}\left\{(-1)^{n-j}-1+2(n-j)\right\}-\sum_{s=3}^{n-j+1} \frac{(-2)^{s-3}}{s}\left(\begin{array}{c}
n-j \\
s-1
\end{array}\right)\right] \\
= & \frac{1}{n-j}\left[g_{n}-g_{j}-\sum_{s=3}^{n-j+1} \frac{(-2)^{s-3}}{s}\left(\begin{array}{c}
n-j \\
s-1
\end{array}\right)\right] \\
= & \frac{1}{n-j} \frac{\sum_{k=g_{j}+1}^{\left\lceil\frac{n}{2}\right\rceil}\left(k-g_{j}\right) \cdot 2}{n-j+1} .
\end{aligned}
$$

Altogether, for each $i \in N$,

$$
t_{i}=-\frac{\sum_{k=g_{i}+1}^{\left\lceil\frac{n}{2}\right\rceil}\left(k-g_{i}\right) \cdot 2}{n-i+1} \theta_{i}+\sum_{j \in P_{i}(d)} \frac{1}{n-j} \frac{\sum_{k=g_{j}+1}^{\left\lceil\frac{n}{2}\right\rceil}\left(k-g_{j}\right) \cdot 2}{n-j+1} \theta_{j}
$$

which coincides the transfer for the maximal transfer rule.

The case when $|N|$ is odd can be proved in a similar way. 
Remark 4: As in Remark 1, the maxiaml rule can be characterized by axioms in Chun (2006a), but modified to be suitable for two server queueing problems.

\section{Concluding remarks}

In this section, we investigate other properties of the minimal transfer rule in the current context and discuss a possible generalization of queueing problems with two servers.

\subsection{Core, Nucleolus, and Shapley value}

As in the one server case, it can easily be shown that our queueing game $v^{M}$ is concave. Also, it can be checked that the allocations assigned by the minimal and the maximal transfer rules belong to the anti-core of the game.

Chun and Hokari (2004) show that the prenucleolus (Schmeidler, 1969) and the Shapley value coincides on the class of queueing problems with one server. Similarly, we can ask whether these two solutions coincide for our problems. As it turns out, they coincide on the class of problems with less than or equal to 4 agents, but not any more for problems with more than 4 agents. Moreover, our games with four agents do not satisfy sufficient conditions of Kar, Mitra, and Mutuswami (2005) for coincidence of the Shapley value and the nucleolus. It would be interesting to investigate the existence of another sufficient condition satisfied by our games with four agents.

\subsection{Queueing problems with multiple servers}

Our analysis on the queueing problem with two parallel servers can be generalized to the problem with an arbitrary number of servers. We conjecture that an agent $i$ 's utility for $\ell$-server problem is

$u\left(g_{i}, t_{i} ; \theta_{i}\right)=-\left(g_{i}-1\right) \theta_{i}+\frac{\sum_{g_{j}^{M}<g_{i}^{M}} g_{j}^{M} \cdot \ell}{d_{i}} \cdot \theta_{i}-\sum_{k \in F_{i}(d)}\left\{\frac{1}{d_{k}-1} \cdot \frac{\sum_{g_{j}^{M}<g_{k}^{M}} g_{j}^{M} \cdot \ell}{d_{k}} \cdot \theta_{k}\right\}$ 
where $g_{i}=\left\lceil\frac{d_{i}}{\ell}\right\rceil$ for each $q_{\ell}=(N, \theta ; \ell)$ and $d \in D\left(q_{\ell}\right)$. However, we could not prove our conjecture due to computational difficulties.

\subsection{Other issues}

This paper leaves many interesting open questions. First, it would be interesting to generalize the problem by assuming that agents need different amounts of processing time. Also, it would be interesting to study an incentive compatible mechanism for the problem as Suijs (1996) for problems with one server. We hope to address these issues in our future research.

\section{References}

[1] Y. Chun, "A pessimistic approach to the queueing problem," Mathematical Social Sciences 51 (2006a), 171-181.

[2] Y. Chun, "No-envy in queuing problems," Economic Theory 29 (2006b), 151-162.

[3] Y. Chun and T. Hokari, "On the coincidence of the Shapley value and the nucleolus in queueing problems," mimeo, Seoul National University, 2004, forthcoming in Seoul Journal of Economics.

[4] R. Dolan, "Incentive mechanisms for priority queueing problems," Bell Journal of Economics 9 (1978), 421-436.

[5] A. Kar, M. Mitra, and S. Mutuswami, "On the coincidence of the prenucleolus and the Shapley value," University of Essex: Economics Discussion Papers 599, 2005.

[6] F. Maniquet, "A characterization of the Shapley value in queueing problems," Journal of Economic Theory 109 (2003), 90-103.

[7] M. Mitra, "Mechanism design in queuing problems," Economic Theory 17 (2001), 277-305. 
[8] M. Mitra, "Achieving the first best in sequencing problems," Review of Economic Design 7 (2002), 75-91.

[9] H. Moulin, "On scheduling fees to prevent merging, splitting and transferring jobs," mimeo, Rice University, 2004, forthcomong in Mathematics of Operations Research.

[10] H. Moulin, "Proportional scheduling, split-proofness, and mergeproofness ," mimeo, Rice University, 2006.

[11] H. Özsoy, "Coordinated splitting in probabilistic scheduling," mimeo, Rice University, 2005.

[12] D. Schmeidler, "The nucleolus of a characteristic function game," SIAM Journal on Applied Mathematics 17 (1969), 1163-1170.

[13] L. S. Shapley, "A value for n-person games," in Contributions to the theory of Games II, Annals of Mathematics Studies No. 28 (H. W. Kuhn and A. W. Tucker, Eds.), pp.307-317, Princeton, NJ:Princeton University Press, 1953.

[14] J. Suijs, "On incentive compatibility and budget balancedness in public decision making," Economic Design 2 (1996), 193-209. 